\title{
Discussion to the papers by R. G. Luke and A. C. Kennedy; and C. R. Blagg
}

GoldSMITH. One can but agree with the contentions of the previous two speakers in their excellent reviews. For this reason I would like to concentrate in this discussion on a few points which are not frequently made in the literature.

The first is in relation to obstructive uropathy. Patients in whom this diagnosis has been missed are still admitted to renal units. The failure to diagnose a post-renal obstruction to the flow of urine must more often be put down to the omission of a thorough rectal and vaginal examination, than to lack of facilities for radioactive renography. It is less well known that in such cases haemo- or peritoneal dialysis are sometimes followed by a copious diuresis. The probable cause is the resorption of oedema at the site of obstruction. This has frequently been the cause of a missed or delayed diagnosis of obstruction. It should also be better known that the ability to pass ureteric catheters to the kidneys does not exclude the diagnosis of obstructive uropathy due to retro-peritoneal fibrosis.

In a small percentage of patients with non-obstructive acute reversible renal failure, its cause remains obscure after full clinical assessment. In this difficult group one should consider the following possibilities: (a) decapitation of a long-standing but previously unrecognized severe hypertension; (b) massive embolization of both, or the only functioning kidney; (c) pancreatitis; and (d) an entity, which for lack of a better name, I here call interstitial nephritis, on account of the biopsy features of widespread interstitial oedema with foci of small round cell infiltrations, a varying degree of tubular atrophy and glomeruli which appear normal on lightmicroscopy. Drug sensitivity, systemic infections such as brucellosis, or renal infections may be causal. The evolution of this type of renal failure may take several weeks. Oliguria is not a constant feature and radiologically, owing to renal oedema, the kidney shows more enlargement than is usually seen in acute reversible renal failure. The urinary urea tends to be higher than in cases of acute tubular necrosis with a corresponding degree of azotaemia. In spite of marked histological changes almost complete functional and histological return to normality has been demonstrated in 1 year follow-up studies.

In contrast to this, nephrotoxic acute renal failure does not always carry as benign a prognosis as has been alleged, if the acute episode is survived. This was demonstrated by one of our patients with severe carbon tetrachloride poisoning who, 1 year later, had a creatinine clearance only $50 \%$ of normal, together with marked tubular atrophy and interstitial fibrosis in a renal biopsy, which was taken for medico-legal purposes.

Factors contributing to the decreasing mortality of acute renal failure include a policy of dialysing such patients before they have become critically ill and dialysing them at shorter intervals. More generous nutrition, firmer fluid restriction and better appreciation of the dangers of over-rapid correction of electrolyte imbalances and anaemia have also helped to increase survival. Blood transfusion need generally not be given until the packed cell volume is below 20-22\%, except in patients with heart disease or where further blood loss or gross haemolysis is anticipated. Transfusions are best given during dialysis, so that excess fluid and potassium can be removed at the time. Infection continues to take its toll in acute renal failure, particularly in posttraumatic cases. Intravenous feeding with fat preparations may, in future, permit increased survival in this $\frac{\bar{\rho}}{\vec{\sigma}}$

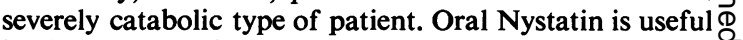
in the prophylaxis of monilial stomatitis and diarrhoea.

The question of peritoneal versus haemodialysis is $\omega$ still discussed. Most workers are agreed that both methods $\overrightarrow{0}$ are applicable in most cases, but that peritoneal dialysis $\overrightarrow{-}$ requires fewer resources and less skill. Very tall, muscular $\vec{\omega}$ or hypercatabolic patients are best treated by haemodialy- $-\Omega$ sis. Except in units with much paediatric experiencee peritoneal dialysis is probably the safer method in babies. 3 . The composition of peritoneal dialysis solutions at present commercially available in this country is not ideal for prolonged maintenance therapy. A solution is required containing a little less sodium, less lactate and $\omega$ some potassium.

The prognostic role of renal needle biopsy in acute renal failure of uncertain aetiology should not be exaggerated. Whilst it is an excellent method of confirming $\vec{\square}$ the diagnosis and potential survival of patients sufferingo from glomerulonephritis, it is unsafe to assume that a specimen showing cortical necrosis necessarily constitutes $\vec{\varphi}$ a death-warrant. This lesion is initially multi-focal and only those patients in whom the lesions become confluent * are doomed.

Finally, with the general availability of peritoneal dialysis, should patients with acute renal failure still be treated only in special units? There are now sufficient renal insufficiency units in the United Kingdom to $\mathbb{D}$ deal with all cases of acute renal failure, at least in peace $\vec{F}$ time. Renal failure carrying a good prognosis, such as $\overline{\overrightarrow{0}}$ that following mis-matched blood transfusion and some obstetric accidents can often be treated successfully by interested personnel in a well-equipped general hospital. However, unexpected complications can arise $\bar{\partial}$ and my preference would be to have every patient 3 treated in a special unit. This applies particularly to post-surgical or post-traumatic renal failure whose 3 . mortality, even in the best hands, approaches or exceeds $\delta$ $50 \%$.

TAYLOR. I have enjoyed greatly the contributions of 0 Dr Luke and Dr Blagg. It is, however, a retrograde step to include pre-renal and post-renal conditions within $\frac{7}{2}$ the definition of acute renal failure. Clinicians and biochemists have learned to differentiate, on the basis of $\mathrm{N}$ urea excretion, between the oliguria or raised blood urea concentration caused by intrinsic renal damage and that $\mathrm{N}$ caused by pre- or post-renal factors. They know, too, $\mathrm{N}$ that the treatment of intrinsic renal failure is quite $\sigma$ different from that of the extrarenal uraemias, so that great therapeutic harm might result from a confused nomenclature. A term like 'post-renal acute renal failure' is thoroughly clumsy as well as being untrue, for the : kidneys may not have failed. How much more elegant is the 'obstructive uropathy' of Dr Goldsmith.

My experience of the use of U/P osmolality ratios in order to differentiate between intrinsic renal failure and $\triangle$ the extrarenal uraemias is limited, but $I$ would advise $\sigma$ caution until more information is available. Osmolality 
is one measure of all the particles in a solution, specific gravity is another. The two are therefore not unrelated, yet the latter has been widely and rightly abandoned for making this differential diagnosis. Interpretative errors in both may result from the glucosuria and proteinuria that sometimes accompanies trauma as well as from the widely-varying sodium (and chloride) concentrations that Dr Luke has mentioned.

I agree that oliguria is not always present in acute renal failure and was, I think, one of the first to point this out in Britain (Taylor, 1957). In order to diagnose acute renal failure when there is no oliguria, $I$ thus introduced a working definition of acute renal failure as occurring when, with a blood urea of $100 \mathrm{mg} / 100 \mathrm{ml}$ or more, the urinary urea concentration is below $2.0 \mathrm{~g} / 100 \mathrm{ml}$. The physiological justification for this rests upon the work of MacLean \& de Wesselow (1920) who showed that when there is a urea load, the level of urea in the urine should exceed $2 \mathrm{~g} / 100 \mathrm{ml}$ if the kidneys are undamaged. The use of urea $U / P$ ratios in the diagnosis of intrinsic renal damage is advocated by Dr Luke despite the fact that they have been introduced several times in the last $\mathbf{4 0}$ years and have been always abandoned. This is because the linear relationship between urinary and blood urea, that the use of the ratio implies, does not exist, physiologically. The point may be illustrated by examining Dr Luke's recommendation that a U/P urea ratio of less than $14: 1$ is strongly suggestive of the onset of acute renal failure. When the blood urea is $100 \mathrm{mg} /$ $100 \mathrm{ml}$ he would thus fail to diagnose acute renal failure if the urinary urea exceeds $1.4 \mathrm{~g} / 100 \mathrm{ml}$ despite the evidence quoted above that values up to $2.0 \mathrm{~g} / 100 \mathrm{ml}$ are abnormal. There would thus be the risk of missing acute renal failure when the blood urea is at this level and perhaps worse, of treating such a patient with a liberal fluid intake in order to promote a diuresis. On the other hand, when the blood urea is $300 \mathrm{mg} / 100 \mathrm{ml}$, acute renal failure would be diagnosed with urinary urea levels between 2.0 and $4.2 \mathrm{~g} / 100 \mathrm{ml}$. There are no circumstances in which, if words bear their usual meaning, kidneys secreting urea at this concentration can be said to be in 'failure'. Dr Luke's definition must therefore include many patients as having renal failure who show no real evidence of intrinsic renal damage and who, when given mannitol, would show a gratifying diuresis, but who would also respond to oral water. His views upon the usefulness of mannitol must therefore be re-evaluated after adopting a more physiological definition of acute renal failure.

Whether using a urinary level of $2.0 \mathrm{~g} / 100 \mathrm{ml}$ or the urea $U / P$ ratio, or the osmolalities, there are three pitfalls in the diagnosis of acute renal failure of which one must be aware. First, oliguria and/or a rising blood urea obviously occur more readily after trauma or operation when the kidneys are chronically damaged than when there is normal renal function. Of a series of forty-two patients who developed renal failure (acutely), Professor Reid and I found that eleven showed at necropsy moderate or severe antecedent renal damage not recognized during life (Taylor \& Reid, 1958). By the criteria that have been advocated this morning such patients would be diagnosed as having acute renal failure and be treated as such, and indeed acute-upon-chronic failure may have supervened. Yet sometimes such patients have not suffered a further renal insult and may respond to a liberal fluid regime by a diuresis. Evidence of previous renal damage should therefore always be sought. This group of patients would also, incidentally, respond well to mannitol, but would not have been prevented from developing acute failure by its use. Secondly, patients with metabolic alkalosis often show a raised blood urea with a low urinary urea (below $2.0 \mathrm{~g} / 100 \mathrm{ml}$ ). They invariably respond well to a liberal fluid regime (and would to mannitol) and the urinary urea concentration rises quickly. They have not suffered intrinsic renal damage despite the biochemical data. The patient quoted illustratively by Dr Luke this morning is unfortunately just such a patient. Thirdly, if one kidney is infarcted, as may happen after arterial surgery, the remaining kidney will continue to excrete urea in the urine at a concentration above $2.0 \mathrm{~g} / 100 \mathrm{ml}$ but, because only one kidney is active, a free diuresis in response to a liberal fluid regime is difficult to obtain, and fluid overload may ensue.

The diagnosis of intrinsic renal damage in the very early stages after a potential renal insult, before the blood urea reaches $100 \mathrm{mg} / 100 \mathrm{ml}$, remains a very difficult problem. The work of Molloy (1962) seems to be a step in the right direction. He found that a level below $1 \cdot 1 \mathrm{~g} / 100 \mathrm{ml}$ for the urinary urea during the first $24 \mathrm{hr}$ predicted acute tubular necrosis. Looking at our own data in this light the level would seem too low, for several cases would have been missed, but the approach seems worthy of further study.

The behaviour of the urinary sodium in acute renal failure may possibily not be quite so wayward as Dr Luke suggests. The earlier work (Bull, Joekes \& Lowe, 1950) was carried out with the chloride ion before the days of flame photometry. These workers found, invariably, an uncontrolled loss of chloride in the urine in the oliguric and early diuretic phases of acute renal failure so that $\mathbf{P} / \mathbf{U}$ chloride ratios were low, approximately $2: 1$. Professor Read and I were therefore not a little alarmed when we found that our patients invariably had low urinary chloride concentrations with $\mathrm{P} / \mathrm{U}$ chloride ratios usually above $4: 1$. We noted, however, that most of the patients of Professor Bull and his colleagues had acute renal failure of 'non-traumatic' origin. When we divided our patients into 'non-traumatic' and 'traumatic' groups, we found that of ten 'non-traumatic' cases, six had $\mathbf{P} / \mathbf{U}$ chloride ratios of $2: 1$, this agreeing with Professor Bull, whereas in the traumatic group two only of twenty-six patients had ratios of this level. In sixteen the $P / U$ chloride ratios exceeded $4: 1$. It would thus seem that the phase of relative sodium (and chloride) retention which physiologically follows trauma may also operate in the presence of acute renal failure. It would be interesting if Dr Luke were to divide his patients in this way and see if the excretion of sodium shows a similar pattern.

Finally I would like to comment on Dr Blagg's observation that the earlier the dialysis, the better the prognosis, so that he now dialyses patients when the blood urea is in the $200-300 \mathrm{mg} / 100 \mathrm{ml}$ range. My colleague, Dr Lascelles, and I have shown recently (Lascelles \& Taylor, 1966) that if rat brain slices are allowed to respire in oxygen at $37^{\circ}$ with glucose as substrate and that if, after $1 \mathrm{hr}$, urea is added to give a concentration of $400 \mathrm{mg} / 100 \mathrm{ml}$ in the surrounding medium, the oxygen uptake of the slices remains 
unchanged during the 2 nd and 3rd hours. But during the 4th hour, the oxygen uptake falls significantly. Thus in vitro there is an inhibitory effect of urea, but it is delayed. This observation would explain why in acute experiments in animals and man, urea is found to be without toxicity. In clinical uraemia this delayed inhibitory effect might perhaps occur with lower urea levels after 1 or 2 days, in which case the beneficial effects of early dialysis would be explained more precisely.

\section{References}

Bull, G.M., Joekes, A.M. \& Lowe, K.G. (1950) Renal function studies in acute tubular necrosis. Clin. Sci. 9, 379.

LASCELlES, P.T. \& TAYLOR, W.H. (1966) The effect upon tissue respiration in vitro of metabolites which accumulate in uraemic coma. Clin. Sci. 31, 403.

MacLean, H. \& DE Wesselow, O.L.V. (1920) On the testing of renal efficiency, with observations on the urea coefficient. Brit. J. exp. Path. 1, 53.

Molloy, P.J. (1962) The early diagnosis of impaired postoperative renal function. Lancet, i, 696.

TAYLOR, W.H. (1957) Management of acute renal failure following surgical operation and head injury. Lancet, ii, 703.

TAYLOR, W.H. \& REID, J.V.O. (1958) Acute renal failure after surgical operation and head injury. Brit. J. Surg. 46, 136.

LUKE. In reply to Dr Taylor's points, firstly I would like to agree with him that our terminology for the types of 'acute renal failure' is somewhat inelegant; however we and others (Merrill, 1965) with extensive experience have found it a sound and practical approach when faced with the clinical problem of the oliguric patient with a rising blood urea. There is no doubt that both in pre-renal and post-renal failure there is often a profound diminution in renal blood flow and glomerular filtration rate and hence failure by the kidney to perform its normal functions; the important practical point, which our classification endeavours to highlight, is that the clinician must always consider whether there is any immediate measure, such as replacement of circulating blood volume or relief of ureteric obstruction, which will allow renal function to return towards normal.

As we have emphasized, the differentiation of pre-renal from renal failure is often difficult. No single test can invariably be depended on to give the diagnosis and all tests must be evaluated in the light of the total clinical situation. The level of urine urea achieved during urea loading of a normal subject is not necessarily applicable to the patient with a urea load but with a diminished renal blood flow due to, for example, oligaemia, and our experience suggests that to take a rigid level of $2 \mathbf{g}$ / $100 \mathrm{ml}$ for urine urea as separating pre-renal from renal failure is less useful than consideration of plasma:urine urea and osmolality ratios.

There are, justifiably, arguments as to whether mannitol actually prevents the development of intrinsic renal failure. We believe that it can, and have stated the case for its efficiency. Whether this is accepted or not it should be remembered that mannitol is safe when used in the way we have advocated and that the condition we are trying to prevent carries a substantial mortality.

\section{Reference}

MERRILL, J.P. (1965) The Treatment of Renal Failure, 2nd edn, p. 119. Heinemann Medical Books, London.
Brooks. I agree with Dr Taylor that it is incorrect to place many different causes of oliguria together and call $\stackrel{\varrho}{c}$ them pre-renal failure and add obstructive conditions which like procidentia are sometimes readily reversible. It is unrealistic to call low salt syndrome or cardiac failure, $\overrightarrow{0}$ pre-renal failure; or prostatic obstruction, post-renal $\frac{\bar{\sigma}}{\sigma}$ failure. The kidneys are not primarily involved and the conditions should be called by their proper name. In $\frac{\widehat{\mathbb{D}}}{\mathrm{D}}$

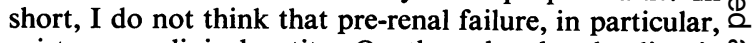
exists as a clinical entity. On the other hand, oliguria after major surgery, especially open heart surgery, is $\mathbf{a} \overrightarrow{0}$ common finding but only very rarely does it develop into the distinct clinical and pathological entity we call acute $\overrightarrow{\vec{\omega}}$ renal failure.

Progress will not be made in preventing acute renal failure if we infuse an osmotic diuretic, in a hit-or-miss fashion, into patients with oliguria who would in no circumstances develop renal damage and then believe, $\vec{\omega}$ because we produce a diuresis, we have prevented renal $\overrightarrow{0}$ damage from occurring. There are circumstances where $\$$ mannitol infusions are harmful. For example, pul- $\omega$ monary oedema is sometimes precipitated when there is $N$ pre-existing cardiac failure. When oliguria is due to $\frac{\mathrm{O}}{2}$ dehydration or salt depletion, an osmotic diuretic is contra-indicated because further losses are incurred $D$ during the osmotic diuresis.

I think also it is necessary to put urine specific gravity and urine osmolality into its proper perspective. It $\mathscr{G}$ would be ridiculous, for example, to stop our nurses. making this important clinical measurement routinely. It is true that there may be only a loose relationship between specific gravity and osmolality. Osmolality is a function of particles in solution and therefore upon the degree of ionization of the solute. For example, $\mathrm{NaCl}$ has twice the osmotic activity of urea although the molecular weights of the two substances are very nearly $\overrightarrow{\overrightarrow{0}}$ identical. The specific gravity, on the other hand, is a function of the mass of the particles in solution as well as the number. In this way particles of high molecular weight such as glucose and protein give misleadingly high specific gravities.

Nevertheless, a low urine volume (e.g. less than 500 $\mathrm{ml} / 24 \mathrm{hr}$ ) of low specific gravity (SG less than 1.012) indicates acute renal failure. A low volume urine which has a relatively high $\mathrm{SG}$ requires further evaluation because it may contain materials such as glucose, protein, mannitol, dextran, etc. Also we know that acute 음 renal failure may be present when the urine output $D$ is a litre or more and the SG is low (Sevitt, 1956). Approximate equivalent values for specific gravity and $\overline{\mathrm{N}}$ osmolarity in the diagnosis of renal failure are available $\mathrm{N}$ (Brooks, 1958).

\section{References}

Brooks, D.K. (1958) The modern treatment of anuria and oliguria. Postgrad. med. J. 34, 583.

SEvitT, S. (1956) Distal tubular necrosis with little or no oliguria. J. clin. Path. 9, 12.

LEE. The most important factor to my mind in recognizing acute renal failure is an awareness of the situations during which it may arise. The large number of measurements and ratios of electrolytes or urea suggested as giving an immediate assessment of renal failure point to 
the fact that no single test is adequate. Indeed if one is going to pay attention to these ratios (i.e. those of urine to plasma, urea, sodium, potassium and osmolarity ratios) then it is important to do all the measurements and not to rely on any single test. This is because of the wide range of normality for each test which often overlaps the pathological state. Further, due attention to the state of salt and water balance, other electrolyte balance and the degree of uraemia at the time of the tests is imperative. It appears that, providing adequate fluid replacement has been given, serial measurement of the blood urea is the best single measurement. This provides a fairly adequate test of gauging the onset and continuation of acute renal failure. Even in those situations where hypercatabolic states can be anticipated (surgery, trauma, burns, gastro-intestinal bleeding, infections) - and these same situations may lead to acute ischaemic renal damage - this is still a valuable test, for with normal renal function there is a ceiling level to which the blood urea may rise. The emphasis is on serial measurements of the blood urea. It is fortunate that mannitol is such a harmless agent and often it appears of value. I am also a little concerned at the liberal usage of bladder catheterization that has been proposed when carrying out such treatment, and feel that in the great majority of situations such treatment can be carried out adequately without resort to catheterization.

I think the earlier speakers have been very conservative in their approach to the dietary management of their cases. In non-traumatic cases of acute oliguric renal failure, it is now possible to give these patients a diet containing not less than $2000 \mathrm{cal} /$ day inclusive of 20 $\mathrm{g}$ of first class protein, minimal electrolytes and not more than $500 \mathrm{ml}$ of fluid. Furthermore, such a regime contains a 3-day rotating menu system and patients tolerate it extremely well. This has been a great advance on the severe restrictive regime previously imposed on such patients, not only from a fluid standpoint but from the nature of the caloric intake. I think it is most important to stress that in those cases of hypercatabolic acute renal failure, who will usually need frequent dialysis, preferably haemodialysis, then virtually no restriction of their fluid and dietary intake (high calorie 3000 or more, $40-80 \mathrm{~g}$ of protein) is required. We are all aware of patients who have been repeatedly dialysed, surgically treated and kept in good electrolytic balance throughout their illness and yet they have become progressively emaciated and often died from what is usually called toxaemia. It is in these particular patients that adequate nutrition is most important and not the severe restrictions that one has become so used to. Many of these same patients cannot, for a number of reasons, take food orally and in these situations it is now possible to give them adequate parenteral nutrition. Their frequent dialysis treatments permit of sufficient fluid removal to allow for adequate intravenous therapy.

In regard to the complications of peritoneal and haemodialysis a few comments are appropriate. Whilst if possible it is desirable to put shunts in for acute renal failure cases needing haemodialysis, nevertheless simple cannulation of the radial artery and a corresponding concomitant vein, which can be done by any simple physician not practiced in the art of surgery, provides a means of a very adequate haemodialysis suitable for repeated usage. These same cannulae can be used for taking arterial samples in between dialyses and the venous one used for intravenous therapy, which is often much appreciated by a house physician faced with a patient who has few veins for possible intravenous therapy. This is not desirable when using a shunt.

In peritoneal dialysis I think it is probably unwise to use 2-litre exchanges. This not only causes the patient considerable discomfort but has higher risk of inducing pulmonary complications. By using 1-litre exchange and rapid cycling, an equally efficient dialysis over $24 \mathrm{~h}$ can be effected with rather less discomfort to the patient and, of course, fewer complications. On comparing peritoneal and haemodialysis, I think the question of the disequilibrium syndrome with regards to the latter method has been over-emphasized. It occurs with both types of dialysis and in my own personal experience has been seen more often with peritoneal than with haemodialysis. If peritoneal dialysis is to be used in situations of hypercatabolic acute oliguric renal failure, and I doubt that this is desirable, then one must consider the considerable protein losses that can occur by this route and allow for appropriate replacements preferably by infusions of triple strength plasma. This not only replaces the albumin losses but also replaces the important $\gamma$-globulin losses, remembering that uraemic patients are particularly prone to infection. Whilst it is true that peritoneal dialysis offers many advantages to the doctor, let there be no doubt in anybody's mind that patients who have experienced both peritoneal and haemodialysis, and I personally have conducted a trial on this point, always prefer, without exception, haemodialysis. I think the dogmatic statement, that peritoneal dialysis alone is applicable in paediatrics is very shortsighted and indeed not true. In a recent review of the world literature, which I have just completed, the complication rates for peritoneal and haemodialysis in paediatrics were $14.8 \%$ and $5.9 \%$ respectively. The complication rates for both these procedures are far lower in paediatrics than in adults and are probably a reflection of the far greater individual attention given to paediatric patients than to adult patients. It is important to stress that in paediatric practice both techniques require equally stringent supervision and that in many ways the shorter time of haemodialysis in this situation is an important factor. Undoubtedly both methods have specific application in the paediatric field but in a specialized unit haemodialysis affords rather easier control of the infant's water and electrolyte balance, even in those weighing only $6 \mathrm{lb}$ and being only 6 weeks old. It must be stressed that the haemodynamic alterations that are so often cited as an absolute contraindication to haemodialysis in children can occur just as readily with less chance of early correction in peritoneal dialysis. I am a little dubious of the value of letting peripheral hospitals manage their own cases of acute oliguric renal failure with the help of peritoneal dialysis. It has been my experience to spend much time in putting right the complications of such treatment carried out by the inexperienced. It must also be remembered that any dialysis procedure plays but a small part in the overall management of acute renal failure, and because of this I think that such patients are better treated in specialized units. 
BERLYNE. I would first like to congratulate both $\mathrm{Dr}$ Luke and Dr Blagg on their excellent presentation of a complicated and controversial subject in such a short space of time. I feel obliged to take up Dr Blagg's reference to Resonium A. Resonium A, as you are all aware, is a sodium cycle salt containing approximately $3.2-3.5 \mathrm{mEq}$ of sodium per gram of resin, and its use has been recommended in the past for the treatment of hyperkalaemia in doses of 45-60 g/day by mouth. If one examines faecal cation content after giving sodium cycle resins of the Resonium A type one finds that about $2 \mathrm{mEq}$ of sodium are absorbed per gram of resin administered, and sometimes as high as $3 \mathrm{mEq} / \mathrm{g}$. This makes the sodium absorption from constant dosage of Resonium A, 120-180 mEq/day. This is important in chronic renal failure and in acute renal failure unless one dialyses every day. We tend to dialyse only when indicated and this works out to be once every 10 days using our modified low protein anuric diet. We therefore would risk getting our patients into $1800 \mathrm{mEq}$ of positive sodium balance if we administered Resonium A. On occasions this is fatal and commonly causes heart failure and hypertension with an elevation of the serum sodium level. We therefore introduced a calcium cycle resin, the calcium of which does not appear to have any deleterious effects with this exchange for potassium or other ions in the gut. We would therefore recommend that Calcium Resin as Calcium Zeocarb 225 (obtainable from Messrs Koch-Light Laboratories Ltd, Colnbrook, Bucks, England) be given routinely in the treatment of hyperkalaemia.

An interesting, and we think important, issue in the treatment of hyperkalaemia by resins is the avoidance of the use of sorbitol with the resins. The resins either in the sodium or calcium cycle, pick up potassium in $\mathrm{C}$ circumstances where faecal-water potassium concentra- $\Rightarrow$ tion is highest. As Wrong has shown, faecal-water $\stackrel{\text { ? }}{+}$ potassium concentration is highest in the colon. It wouldo therefore seem logical to keep the resin in the large bowelo for as long as possible. This can be achieved by constipat- $\overline{\bar{c}}$. ing the patient rather than giving him diarrhoea. We have $\widehat{\varnothing}$ done experiments showing that both sodium and calcium cycle resins pick up tiny amounts of potassium during sorbitol diarrhoea, and very much larger amounts in the $\vec{\circ}$ presence of constipation. Sorbitol also causes severe intestinal colic with the passage of foul flatus and oc- $\vec{\omega}$ casional explosive diarrhoea. This seems a high prices to pay for taking a drug which renders the potassium uptake by resin less effective. I would therefore counsel 3 . that sorbitol should not be used with resins. I think its. main use should be in patients where dialysis is to be post- $\omega$ poned and in whom hyperkalaemia is not a problem. $\overrightarrow{0}$ Otherwise, it would be better if the sorbitol were taken by the physician who prescribed it rather than by the unfortunate patient! In this I am supported by the nursing staff on our unit, who loathe sorbitol therapy. $\frac{\text { ? }}{3}$ We would also recommend the use of calcium resins rather than the sodium resins in both acute and chronic $\frac{D}{0}$ renal failure.

One final point is that it has been shown that small $\vec{\bullet}$ fine-ground resins with particles up to $5 \mu$ can get taken $\stackrel{\mathscr{O}}{\stackrel{9}{ }}$ up through the intestinal mucosa and lodge in the brain, liver and other viscera. It seems to me, therefore, that the risk of taking fine-ground resins such as Resonium A is greater than the advantages incurred, unless the patient is sodium depleted or dialysis is being carried out. 